\title{
Conditional convergence of maths and physics
}

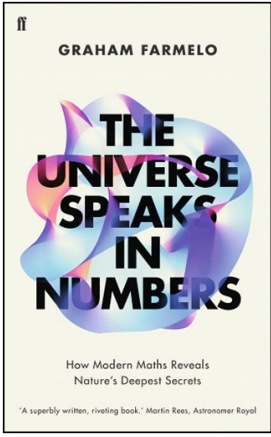

$\mathrm{F}$ or many people - myself included - mathematics can be the most intimidating of the fundamental sciences: an unfathomable language accessible only to a select few with the ability to master its complex myriad of symbols and rules. It is often overshadowed by its close relative physics, as the unpredictable nature of the quantum world, the enigma of a black hole or even just an episode of Star Trek never fails to capture the public's imagination. It seems that mathematics never receives the credit it deserves.

Enter The Universe Speaks in Numbers by Graham Farmelo, which aims to bring mathematics to the forefront of the reader's mind and convince them that mathematics has and will continue to play an indispensable role in the future of physics. From the writings of the ancient Greeks through to the latest developments at the Large Hadron Collider, the book aims to highlight the invaluable contribution of mathematics to the most important advances scientists have made in uncovering the fundamental laws that govern the Universe.

By immersing us in historic details the book narrates the circumstances of key discoveries and the individuals behind them. It begins with Newton's Principia and the foundation of classical mechanics, Maxwell's work on electromagnetism, Einstein's theory of general relativity through to quantum mechanics. Each field is exemplified to showcase how the research, although firmly embedded in the world of physics, was also based on foundations of profound mathematical insight. A wide range of wellknown scientists is brought in to provide a narrative of the surrounding circumstances, differing views, seminal lectures and publications, as well as to illuminate how the relationship between theoretical physics and

mathematics has evolved through the centuries.

Farmelo laments the fact that, despite joint successes, mathematicians and theoretical physicists have tended to take separate paths, sometimes pouring scorn on each other along the way, and that too few scientists, even now, have sought to establish themselves in both fields. The book describes how influential figures - and even a clandestine, masonic-like mathematical society publishing under the pseudonym of Nicolas Bourbaki - aimed to drive a wedge between the two fields. This was an era described as the 'long divorce' in an interview with the renowned theoretical physicist and mathematician Freeman Dyson. According to Dyson, despite fruitful collaborations between the two fields, the two began to diverge approaching the Second World War. Physicists became content adopting the mathematical tools already available for new theories and mathematicians preferred to focus on the beauty of their equations with little care for any potential relevance to the real world.

This period came to an end during the 1970s with the inception of string theory: a theoretical framework that some believe is the strongest candidate for a unified theory that encompasses all four fundamental forces. However, to test this in an experiment, string theory requires energies and distances on a scale that today's researchers are more likely to see in Star Trek than in the most advanced particle accelerator. Here, Farmelo argues that 'orthodox' methods such as observation and experiment cannot be relied upon, and so instead theoretical physicists should "use the torch of mathematics to help light the way forward" until, eventually, technology catches up to test theories experimentally.

This touches upon one of the biggest dilemmas facing the current generation of theoretical physicists: that the flow of experimental data in need of explanatory theories has slowed. The vast amount of data collected at the Large Hadron Collider has not discriminated between theories in the way that particle physicists would have hoped for and in such circumstances Farmelo argues that one has to be more creative, rely on pure thought and adapt abstract mathematical concepts to develop new theories. This is a methodology termed 'physical mathematics' and described as: "A fusion of mathematics and theoretical physics that aims to shed light on new mathematics, on the fundamental laws of Nature, and on the relationship between them."

This suggestion is not without controversy, since some see this subfield as an unhelpful distraction for physicists who could be swallowed up in a mire of mathematical trivialities.

The book's structure is reminiscent of Brian Greene's The Elegant Universe that also sought to encourage enthusiasm for string theory. Both authors first set the scene by describing general relativity and quantum mechanics, but from there Greene's approach is more education-based, seeking to help the reader grasp basic concepts by employing imaginative although sometimes rather complex thought experiments. In contrast, Farmelo provides a detailed and enjoyable historical overview of key players, focusing just as much on the quirks and flaws of their personalities as well as on their scientific contributions. The book is entertaining and written with a contagious enthusiasm, and could almost be described as a page turner. Indeed, some specifics seem to be included to purely entertain rather than persuade. For example, is it really necessary to inform us that Newton was a virgin upon his death? However, this is minimal and does not distract from the book's main message.

In drumming up enthusiasm for mathematics and its historical importance, the book is a success. Farmelo paints a detailed picture of events, circumstances, relationships and personalities that captures the imagination as well as educates those unfamiliar with this world. But whether it will convince the reader that experimental data can be supplanted by pure mathematics as a guiding principle for theoretical insight is less certain.

There are solid arguments on both sides of the divide, and depending on their experience, people are likely to already have entrenched views on the subject. Therefore, while The Universe Speaks in Numbers is entertaining and informative, I'm not sure how successful it will be in convincing readers to speak to the Universe in its own language.

Reviewed by Saleem Denholme Associate Editor, Communications Physics. e-mail: saleem.denholme@nature.com

Published online: 3 September 2019 https://doi.org/10.1038/s41567-019-0654-X 